Bull. Austral. Math. Soc.

VOL. 48 (1993) [195-200]

\title{
ELLIPTIC INTEGRALS AND LIMIT CYCLES
}

\author{
A.M. Urbina, M. León de la Barra, G. León de la Barra and M. Cañas
}

By using zeros of elliptic integrals we establish an upper bound for the number of limit cycles that emerge from the period annulus of the Hamiltonian $X_{H}$ in the system $X_{c}=X_{H}+\varepsilon(P, Q)$, where $H=y^{2}+x^{4}$ and $P, Q$ are polynomials in $x, y$, as a function of the degrees of $P$ and $Q$. In particular, if $(P, Q)=\left(\sum_{i=2}^{N} a_{i} x^{i}, 0\right)$ with $N=2 k+1$ or $2 k+2$, this upper bound is $k-1$.

\section{INTRODUCTION}

Since the source work of Poincaré [2] polynomial vector fields have received great attention.

In this context, Hilbert's famous $16^{\text {th }}$ problem, concerning the maximum number and positions of Poincaré boundary cycles (that is, limit cycles) for polynomial differential equations of the first order and degree remains open even for the case of quadratic differential equations.

In this work we consider a family of polynomial vector fields $X_{\varepsilon}=X_{H}+\varepsilon(P, Q)$ where $X_{0}=X_{H}$ is the Hamiltonian field corresponding to the Hamiltonian function $H(x, y)=y^{2}+x^{4}$.

By using zeros of elliptic integrals (Theorem 1) we establish an upper bound for the number of limit cycles that emerge from the period annulus of $X_{H}$ as a function of the degrees of $P$ and $Q$.

Next, we apply this result to a related family of Lienard's equation $X_{\varepsilon}(x, y)=$ $X_{H}+\varepsilon\left(\sum_{i=2}^{N} a_{i} x^{i}, 0\right)$ and we obtain for $N=2 k+1,2 k+2$, that at most $k-1$ limit cycles emerge from the periodic trajectories of $X_{0}$ (Theorem 2).

Elsewhere [7] we studied this Lienard's equation and we proved that there is $\tilde{\varepsilon}>0$ such that for $0 \leqslant \varepsilon \leqslant \widetilde{\varepsilon}, X_{\varepsilon}(x, y)$ has no separatrices in a neighbourhood of the origin and we found an upper bound for the maximum number of small amplitude limit cycles that emerge from the origin under the above perturbation of $X_{H}$.

Received 3rd September, 1992

Partially supported by FONDECYT, under grant \# 0243-91 and U.T.F.S.M., under grant \# 92.12.04.

Copyright Clearance Centre, Inc. Serial-fee code: 0004-9729/93 \$A2.00+0.00. 


\section{MELNIKOV'S DEVIATION}

Let $X_{\epsilon}=X_{H}+\varepsilon(P, Q)$ be a one parameter family of vector fields, where $X_{0}=$ $X_{H}$ is a Hamiltonian vector field corresponding to $H(x, y)=y^{2}+x^{4}$ and $P, Q$ are polynomials in $x$ and $y$.

Let $\left.\sum=\right] 0, s\left[\times\{0\}\right.$ be a transversal section to the vector field $X_{0}$.

Melnikov's deviation for the family $X_{\varepsilon}$, with respect to $\sum$ is:

$$
d(x, \varepsilon)=h(x, \varepsilon)-x, \quad x \in \sum
$$

for $\varepsilon$ small enough and $h$ the Poincaré return map.

The zeros of $d(-, \varepsilon)$ correspond to the periodic orbits of $X_{\varepsilon}$ which intersect $\sum$.

If $(\partial d / \partial \varepsilon)\left(x_{0}, 0\right)=0$ and $\left(\partial^{2} d / \partial x \partial \varepsilon\right)\left(x_{0}, 0\right) \neq 0$ then a corollary of the Implicit Function Theorem implies the existence of $\bar{\varepsilon}>0$ and a unique smooth function $\beta:]-\bar{\varepsilon}, \bar{\varepsilon}\left[\longrightarrow \sum\right.$ such that $\beta(0)=x_{0}$ and $d(\beta(\varepsilon), \varepsilon)=0$ for all $\left.\varepsilon \in\right]-\bar{\varepsilon}, \bar{\varepsilon}[$. Moreover, if $x_{0}$ is a simple zero, then $\beta(\varepsilon)$ is also a simple root of $d(x, \varepsilon)=0$ (Bifurcation Lemma, $[\mathbf{1}])$.

We use the normalised displacement function for $X_{0}$ :

$$
F(x, \varepsilon)=4 x^{3} d(x, \varepsilon)
$$

Obviously, for $x \neq 0, d(\beta(\varepsilon), \varepsilon)=0$ if and only if $F(\beta(\varepsilon), \varepsilon)=0$.

The derivative $(\partial F / \partial \varepsilon)(x, \varepsilon)$ has an integral representation (Melnikov's integral [1]) given in our case by $(\partial F / \partial \varepsilon)(x, 0)=\int_{0}^{T(x, 0)}\left(2 y Q+4 x^{3} P\right) d t$, where $T(x, 0)$ is the minimum positive $t$ required for the trajectory $\Gamma$ starting at $(x, 0)$ to return to $\sum$, $x=x(\gamma(t)), y=y(\gamma(t))$ and $\gamma(t)$ is the integral curve corresponding to $\Gamma$.

The above integral is an elliptic integral in Cartesian coordinates.

Several authors have done interesting research on the zeros of this type of elliptic integral with respect to some specific Hamiltonian functions $H[3,4,5,6,8]$.

We proceed now to establish some results on elliptic integrals related to our case .

\section{ZEROS OF ELLIPTIC INTEGRALS}

Let $\Lambda^{1}$ be the space of polynomial forms of order 1 , that is,

$$
\Lambda^{1}=\{\omega=P(x, y) d x+Q(x, y) d y \mid P, Q \text { polynomials }\}
$$

In $\Lambda^{1}$ the following equivalence relation is defined:

$\omega_{1} \sim \omega_{2} \Leftrightarrow \omega_{1}-\omega_{2}=A d H+d B$ where $A, B$ are polynomials in two variables $x$ and $y$ and $H(x, y)=y^{2}+x^{4}$. 
Let $\Omega=\Lambda^{1} / \sim$ be the quotient space and let $[\omega]$ denote the equivalence class of the $1-$ form $\omega$.

There is a natural modulus structure on $\mathbb{R}[h]$ given by

$$
P(h)[\omega]=[P(H) \omega]
$$

with $P$ a polynomial.

It is easily shown that $\omega_{1} \sim \omega_{2}$ implies $\int_{H=h} \omega_{1}=\int_{H=h} \omega_{2}$.

LEMma 1. The classes of the forms $\omega_{j}=x^{j} y d x, j=0,1,2$ generate the modulus $\Omega$.

Proof :

(i) It is clear that

$$
\left[x^{k} y^{2 \ell} d x\right]=\left[x^{4 k} y^{\ell} d y\right]=\left[x^{k} y^{2 \ell+1} d y\right]=0 \quad \forall k, \ell \in \mathbb{N}_{0} .
$$

(ii) The following relation is easily proved by induction on $k, \ell \in \mathbb{N}_{0}$

$$
\left[x^{4 k+j} y^{2 \ell+1} d x\right]= \begin{cases}\sum_{i=0}^{\ell}(-1)^{\prime} \prod_{i=1}^{k+\ell} \frac{j+1+4(i-1)}{j+7+4(i-1)} h^{k+\ell}\left[\omega_{j}\right] & j=0,1,2 \\ 0 & j=3\end{cases}
$$

if $k+\ell>0$.

(iii)

$$
\left[x^{4 k+j} y^{2 \ell} d y\right]=K h^{k+\ell}\left[\omega_{j-1}\right] \quad \forall k, \ell \in \mathbb{N}_{0} \text { and } j=1,2,3,
$$

where

$$
K=-\frac{4 k+j}{2 \ell+1} \sum_{i=0}^{\ell}(-1)^{\ell} \prod_{i=1}^{k+\ell} \frac{j+4(i-1)}{j+6+4(i-1)} \text { if } k+\ell>0
$$

and $K=-j$ if $k+\ell=0$.

This case follows from

$$
x^{4 k+j} y^{2 \ell} d y=d\left(\frac{x^{4 k+j} y^{2 \ell+1}}{2 \ell+1}\right)-\frac{4 k+j}{2 \ell+1} x^{4 k+j-1} y^{2 \ell+1} d x
$$

and (ii).

Lemma 2. For $\omega \in \Lambda^{1}$ and degree $\omega=n$, we have

$$
[\omega]=P_{0}(h)\left[\omega_{0}\right]+P_{1}(h)\left[\omega_{1}\right]+P_{2}(h)\left[\omega_{2}\right]
$$

where

$$
P_{i}(h) \in \mathbb{R}[h] \quad i=0,1,2, \operatorname{deg} P_{0} \leqslant\left[\frac{n}{2}\right], \operatorname{deg} P_{i} \leqslant\left[\frac{n}{2}\right]-1, \quad i=1,2 .
$$


PROOF: Let $\omega=\left(\sum_{i+j=0}^{n} a_{i j} x^{i} y^{j}\right) d x+\left(\sum_{i+j=0}^{n} b_{i j} x^{i} y^{j}\right) d y$. By Lemma 1 ,

$$
[\omega]=P_{0}(h)\left[\omega_{0}\right]+P_{1}(h)\left[\omega_{1}\right]+P_{2}(h)\left[\omega_{2}\right] .
$$

In order to estimate the degrees of the $P_{i}(h) i=0,1,2$ it is enough to calculate the coordinates of the classes $\left[y^{n} d x\right],\left[x^{2} y^{n-2} d x\right],\left[x y^{n-2} d x\right]$ if $n$ is odd, and $\left[x y^{n-1} d x\right],\left[y^{n-1} d x\right],\left[x^{2} y^{n-3} d x\right]$ if $n$ is even, with respect to the basis $\left\{\left[\omega_{0}\right],\left[\omega_{1}\right]\left[\omega_{2}\right]\right\}$.

Let us consider now the real functions

$$
I_{i}(h)=\int_{H=h} \omega_{i}, i=0,1,2
$$

The following relations are easily proved:

LEMMA 3. If $h \geqslant 0$ then

$$
I_{1} \equiv 0 ; \quad I_{0}(h)=\frac{4}{3} h I_{0}^{\prime}(h) ; \quad I_{2}(h)=\frac{4}{5} h I_{2}^{\prime}(h) .
$$

THEOREM 1. Let $\omega \in \Lambda^{1}$ be an n-degree form and $h \geqslant 0$. Then the number of positive zeros of the function $I_{\omega}(h)=\int_{H=h} \omega$ is at most $2 \cdot[n / 2]$

Proof :

$$
I_{\omega}(h)=P_{0}(h) I_{0}(h)+P_{1}(h) I_{1}(h)+P_{2}(h) I_{2}(h) .
$$

From Lemma 3 we obtain $I_{0}(h)=c_{0} h^{3 / 4} ; I_{2}(h)=c_{2} h^{5 / 4} \quad c_{0}, c_{2}>0$. Then $I_{\omega}(h)=$ $h^{3 / 4}\left(c_{0} P_{0}(h)+c_{2} h^{1 / 2} P_{2}(h)\right)$. with $\operatorname{deg} P_{0}(h) \leqslant[n / 2]$ and $\operatorname{deg} P_{2}(h) \leqslant[n / 2]-1$ and the theorem follows.

COROLlary. The maximum number of periodic trajectories in the period annulus of $X_{H}$ at which a continuous family of limit cycles emerges in the system $X_{\varepsilon}=X_{H}+\varepsilon(P, Q)$, where $H(x, y)=y^{2}+x^{4}$ and $P, Q$ are polynomials is at most $2[n / 2]$, with $n=\max \{\operatorname{deg} P, \operatorname{deg} Q\}$.

\section{LIMIT CYCLES OF SOME LIÉNARD'S EQUATIONS}

Let now $X_{\varepsilon}(x, y)=X_{H}(x, y)+\varepsilon\left(\sum_{i=2}^{N} a_{i} x^{i}, 0\right)$ with $H=y^{2}+x^{4}$, be the family corresponding to Liénard's equations $\ddot{x}=\varepsilon f(x) \dot{x}-4 x^{3}$, where $\sum_{i=2}^{N} a_{i} x^{i}=\int_{0}^{x} f(u) d u$.

We have proved that for $\varepsilon$ small enough this family has no separatrices in a neighbourhood of the origin and if $a_{i}=0$ for all $i$ odd, $X_{\varepsilon}$ has a centre at the origin. We have established the maximum number of small amplitude limit cycles that bifurcate 
from the origin in term of $N$ and if the coefficients $a_{i}, i$ odd, satisfy certain relations, this maximun is attained. (Moreover, we have generalised these results for $H=y^{2}+x^{2 n}$ [7].)

Now we prove:

THEOREM 2. The maximun number of periodic orbits in the period annulus of $X_{H}$ at which a continuous family of limit cycles emerges in the system $X_{\epsilon}$ is $k-$ 1 for $N=2 k+1,2 k+2$.

PROOF:

$$
\begin{aligned}
\frac{\partial F}{\partial \varepsilon}(x, 0) & =-\int_{H=h} \sum_{i=2}^{N} a_{i} x^{i} d y=P_{0}(h) I_{0}+P_{2}(h) I_{2} \\
& =h^{3 / 4}\left(c_{0} P_{0}(h)+c_{2} h^{1 / 2} P_{2}(h)\right) \quad c_{0}, c_{2}>0
\end{aligned}
$$

where, for $k$ even we have:

$$
\begin{aligned}
& P_{0}(h)=\sum_{\ell=1}^{k / 2}(4 \ell+1) a_{4 \ell+1} \prod_{i=1}^{\ell} \frac{1+4(i-1)}{7+4(i-1)} h^{\ell} \\
& P_{2}(h)=3 a_{3}+\sum_{\ell=2}^{k / 2}(4 \ell-1) a_{4 \ell-1} \prod_{i=1}^{\ell-1} \frac{3+4(i-1)}{9+4(i-1)} h^{\ell-1},
\end{aligned}
$$

and for $k$ odd

$$
\begin{aligned}
& P_{0}(h)=\sum_{\ell=1}^{(k-1) / 2}(4 \ell+1) a_{4 \ell+1} \prod_{i=1}^{\ell} \frac{1+4(i-1)}{7+4(i-1)} h^{\ell} \\
& P_{2}(h)=3 a_{3}+\sum_{\ell=2}^{(k+1) / 2}(4 \ell-1) a_{4 \ell-1} \prod_{i=1}^{\ell-1} \frac{3+4(i-1)}{9+4(i-1)} h^{\ell-1} .
\end{aligned}
$$

If we make the substitution $h=z^{2}$ then $(\partial F / \partial \varepsilon)(x, 0)=z^{3 / 2} Q(z)$ where $1 \leqslant$ $\operatorname{deg} Q \leqslant k$ in both cases and $(\partial F / \partial \varepsilon)(x, 0)$ has at most $k-1$ positive zeros.

\section{REFERENCES}

[1] C. Chicone and M. Jacobs, 'Bifurcation of limit cycles from quadratic isochrones', $J$. Differential Equations 91 (1991), 268-326.

[2] H. Poincaré, 'Sur les courbes définies par une equation differéntielle', J. Mathematique 7 (1881), 375-422.

[3] G.S. Petrov, 'Number of zeros of complete elliptic integrals', Functional Anal. Appl. 18 (1984), 148-150. 
[4] G.S. Petrov, 'Elliptic integrals and their monoscillation', Functional Anal. Appl. 20 (1986), 37-40.

[5] G.S. Petrov, 'Complex zeros of an elliptic integral', Functional Anal. Appl. 21 (1987), 247-248.

[6] C. Rosseau and H..Zoladek, 'Zeros of Complete Elliptic Integrals for 1:2 Resonance', $J$. Differential Equations 94 (1991), 41-54.

[7] A. Urbina, G. León de la Barra, M. León de la Barra and M. Cañas, 'Limit cycles of Liénard equations with not linear damping', Canad. Math. Bull. (to appear).

[8] A.N. Varchenko, 'Estimate of the number of zeros of abelian integrals depending on parameters and limit cycles', Functional Anal. Appl. 18 (1984), 98-108.

Universidad Técnica Federico Santa María

Departamento de Matemática

Casilla 110 - V

Valparaiso

Chile 